\title{
Immunohistochemical validation of COL3A1, GPR158 and PITHD1 as prognostic biomarkers in early-stage ovarian carcinomas
}

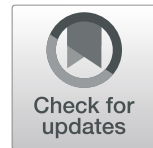

\author{
Hanna Engqvist ${ }^{1 *} \mathbb{D}$, Toshima Z. Parris ${ }^{1}$, Anikó Kovács ${ }^{2}$, Szilárd Nemes ${ }^{3}$, Elisabeth Werner Rönnerman ${ }^{1,2}$, \\ Shahin De Lara ${ }^{2}$, Jana Biermann ${ }^{1}$, Karin Sundfeldt ${ }^{4}$, Per Karlsson ${ }^{1+}$ and Khalil Helou ${ }^{1+}$
}

\begin{abstract}
Background: Ovarian cancer is the main cause of gynecological cancer-associated death. However, 5-year survival rates differ dramatically between the five main ovarian carcinoma histotypes. Therefore, we need to have a better understanding of the mechanisms that promote histotype-specific ovarian carcinogenesis and identify novel prognostic biomarkers.

Methods: Here, we evaluated the prognostic role of 29 genes for early-stage (I and II) ovarian carcinomas ( $n=206)$ using immunohistochemistry (IHC).

Results: We provide evidence of aberrant protein expression patterns for Collagen type III alpha 1 chain (COL3A1), G protein-coupled receptor 158 (GPR158) and PITH domain containing 1 (PITHD1). Kaplan-Meier survival analysis revealed that COL3A1 expression was associated with shorter overall survival in the four major histotypes of epithelial ovarian carcinoma patients ( $P$ value $=0.026, \mathrm{HR}=2.99$ (95\% Cl 1.089-8.19)). Furthermore, GPR158 and PITHD1 were shown to be histotype-specific prognostic biomarkers, with elevated GPR158 expression patterns in mucinous ovarian carcinoma patients with unfavorable overall survival ( $P$ value $=0.00043, \mathrm{HR}=6.13(95 \% \mathrm{Cl} 1.98-18.98)$ ), and an association with lower PITHD1 protein expression and unfavorable overall and disease-specific survival in clearcell ovarian carcinoma patients ( $P$ value $=0.012, \mathrm{HR}=0.22(95 \% \mathrm{Cl} 0.058-0.80)$; $P$ value $=0.003, \mathrm{HR}=0.17$ (95\% Cl 0.043-0.64)).
\end{abstract}

Conclusions: The novel biomarkers identified here may improve prognostication at the time of diagnosis and may assist in the development of future individualized therapeutic strategies for ovarian carcinoma patients.

Keywords: Ovarian cancer, Prognostic biomarker, Immunohistochemistry, Mucinous ovarian cancer, Clear-cell ovarian cancer

\section{Background}

Ovarian cancer is the most lethal gynecological cancer with a five-year survival rate of about $55 \%$ in Sweden and $47 \%$ in the US [1,2]. Epithelial cancers account for about $90 \%$ of all ovarian cancers and are distributed over the most common histotypes: high-grade serous (HGSC, $70 \%$ ), low-grade serous (LGSC, $<5 \%$ ), endometrioid (EC, $10 \%$ ), mucinous (MC, 3-4\%) and clear-cell ovarian

\footnotetext{
* Correspondence: hanna.engqvist@gu.se

${ }^{\dagger}$ Per Karlsson and Khalil Helou contributed equally to this work.

'Department of Oncology, Institute of Clinical Sciences, Sahlgrenska Cancer

Center, Sahlgrenska Academy at University of Gothenburg, Gothenburg,

Sweden

Full list of author information is available at the end of the article
}

carcinomas (CCC, 10\%) [3]. Five-year survival rates differ significantly across the histotypes, with drastically lower survival rates for serous carcinoma (SC (HGSC and LGSC), 43\%) compared to EC (82\%), MC (71\%) and CCC $(66 \%)$ in the US. This is in line with the high number of patients $(80 \%)$ with SC that are diagnosed at advanced stages (stages III and IV) as well as EC, MC and CCC that are predominantly diagnosed at stage I (58-64\%) [4]. Hence, in view of the diverse survival rates for the different histotypes, it is crucial to identify novel prognostic biomarkers for each histotype.

Cancer antigen 125 (CA 125) is routinely used in the clinic for, e.g. preoperative diagnosis, to monitor response

(c) The Author(s). 2019 Open Access This article is distributed under the terms of the Creative Commons Attribution 4.0 International License (http://creativecommons.org/licenses/by/4.0/), which permits unrestricted use, distribution, and reproduction in any medium, provided you give appropriate credit to the original author(s) and the source, provide a link to the Creative Commons license, and indicate if changes were made. The Creative Commons Public Domain Dedication waiver (http://creativecommons.org/publicdomain/zero/1.0/) applies to the data made available in this article, unless otherwise stated. 
to chemotherapy, disease progression and relapse of epithelial ovarian cancer [5]. However, it may not be suitable for the detection of early-stage ovarian cancer nor the MC histotype [5-7]. Additional serum biomarkers, such as CA 19-9, CA 15-3, CA 72-4 and CEA are also routinely used in clinical practice [8]. Biomarker panels, such as CA 125 in combination with human epididymis protein 4 (HE4) (Risk of Ovarian Malignancy Algorithm, ROMA) and the multivariate index assay (MIA2G) comprising CA 125, transferrin, apolipoprotein A-1, follicle-stimulating hormone and HE4 (Overa), have been approved by the US Food and Drug Administration (FDA) for preoperative testing to determine the likelihood of malignancy $[9,10]$. Apart from the use of PARP inhibitors, no prognostic biomarkers for the management of ovarian cancer are currently used in the clinic. Some recent reports have evaluated prognosis in relation to histotype, e.g. the expression of cancer antigen 45 (CT 45) was shown to be a prognostic factor for positive response to platinum-based chemotherapy in HGSC [11]. Furthermore, high expression of aquaporin-1 (AQP1) in MC and EC, and low expression of AQP1 in CCC were associated with unfavorable prognosis [12].

Early-stage epithelial ovarian cancers generally have a more favorable prognosis with an overall five-year survival rate of $89 \%$ for stage I and $71 \%$ for stage II [4]. Yet around $16 \%$ of stage I and II ovarian cancer patients have worse prognoses [4]. Hence, it is important to identify novel biomarkers for use in ovarian cancer prognostication to aid in the management of ovarian cancer in order to identify patients with aggressive disease. In view of differences between the histotypes, histotype-based gene panels for prognosis are also needed to guide therapeutic decisions. In the current study, immunohistochemistry (IHC) was used to evaluate the clinical relevance of 29 promising prognostic biomarkers (ARHGAP21, ARMC3, C7, CDH18, CES3, COL11A1, COL3A1, EHD3, FRMPD2, GABRP, GID4, GPR158, GRM5, IGHG1, JCHAIN, KIF26B, MAP7D2, MTRNR2L1, MTUS1, MUC15, PITHD1, PTEN, RTKN2, SLC9A4, SMYD2, TRIM71, TRIO, TTK, and VNN1) for early-stage ovarian carcinoma identified using RNA sequencing (RNA-seq) data. In our previous work, MTUS1 frameshift insertion was associated with differences in gene expression and overall survival, and COL3A1 gene expression were shown to correlate with tumor aggressiveness [13]. The remaining 27 biomarkers were identified using Cox regression models to correlate gene expression data (RNA-seq) with survival status.

\section{Methods}

\section{Patients and tumor samples}

Full-face formalin-fixed paraffin-embedded (FFPE) specimens were obtained from the Departments of Clinical Pathology at hospitals in Western Sweden for 206 early- stage (stage I and II) primary invasive ovarian carcinoma patients diagnosed between 1994 and 2006, of which 95 samples corresponded to fresh-frozen tumor samples previously analyzed by RNA-seq [13]. All tumor specimens were reclassified according to current WHO criteria [14-17] by pathologists at Sahlgrenska University Hospital. Further clinicopathological characteristics data were obtained from the National Quality Registry at the Regional Cancer Center West (Gothenburg, Sweden) and the Cancer Registry at the National Board of Health and Welfare (Table 1). All procedures were performed in accordance with the Declaration of Helsinki and approved by the Regional Ethical Review Board (Gothenburg, Sweden; case number 767-14). The Regional Ethical Review Board approved a waiver of written consent to use the tumor specimens.

\section{Selection of study genes}

Raw RNA-seq read counts (log2-values) for 95 of the 206 ovarian tumors were used to correlate gene expression data with survival status (overall survival (OS) and disease-specific survival (DSS)) for each histotype (HGSC, EC, MC, CCC) using univariable Cox proportional hazards models and the Benjamini-Hochberg procedure to control the false discovery rate (FDR) with FDR-corrected $P$ values $<0.05$ [13]. The Cox regression analysis assumes the following relationship between the baseline and observed hazard $h_{1}(t)=h_{o}(t) e^{\Sigma \beta x}$. Here, $e^{\beta x}$ quantifies the effect of individual probes on the baseline hazard. Using the output from the Cox regression analysis, an index was defined as $E\{x \mid \beta x>0\}-E\{x \mid \beta x<0\}$, wherein $E$ is the expected value. The index assessed the absolute difference in mean $\log 2$ ratio of different probes among patients with favorable $\beta x<0$ and unfavorable $\beta x>0$ prognoses. The predictive power of the regression models was assessed with time-dependent Area under the receiver operating characteristics curve $[(\mathrm{AUC}(\mathrm{t})]$ values and summarized as the concordance index (C-index) for survival data [18]. The C-index varies between 0.5 (random ordering of the survival time with no predictive power) and 1 (perfect ordering of the survival times, i.e. perfect prediction of survival times). Twenty-seven genes, henceforth termed study genes, were selected among genes with $P$ values below 0.05 and the highest $\mathrm{C}$-index and/or the highest absolute $\log 2$ ratio value.

\section{Immunohistochemical analysis}

Four micrometer FFPE sections were prepared on Dako FLEX IHC microscope slides and dried in an oven for 1 hour at $60^{\circ} \mathrm{C}$. Antibodies for the study proteins were primarily chosen from the Human Protein Atlas (HPA) $[19,20]$. Optimal antibody dilutions were achieved using an optimization panel consisting of 15 full-face FFPE ovarian carcinoma sections representing varying 
Table 1 Clinicopathological characteristics for the 206 ovarian carcinoma patients

\begin{tabular}{|c|c|c|c|c|c|}
\hline & \multicolumn{4}{|c|}{ Number of patients (\%) } & \multirow[b]{3}{*}{$P$ value } \\
\hline & HGSC & EC & $M C$ & $\mathrm{CCC}$ & \\
\hline & $(n=94)$ & $(n=46)$ & $(n=29)$ & $(n=37)$ & \\
\hline Patient age & & & & & 0.457 \\
\hline Mean & 64 & 62 & 60 & 65 & \\
\hline Range & $22-88$ & $25-83$ & $30-82$ & $42-84$ & \\
\hline Overall Survival & & & & & 0.082 \\
\hline $0-2 y$ & $7(7)$ & $3(7)$ & $6(21)$ & $5(14)$ & \\
\hline $2-5 y$ & $26(28)$ & $9(20)$ & $3(10)$ & $10(27)$ & \\
\hline $5-10 y$ & $28(30)$ & $7(15)$ & $7(24)$ & $8(22)$ & \\
\hline$>10 y$ & $33(35)$ & $27(59)$ & $13(45)$ & $14(38)$ & \\
\hline Cause of death & & & & & 0.001 \\
\hline Ovarian carcinoma & $53(56)$ & $7(15)$ & $5(17)$ & $19(51)$ & \\
\hline Other cancer & $8(9)$ & $6(13)$ & $4(14)$ & $2(5)$ & \\
\hline Other & $10(11)$ & $10(22)$ & $8(28)$ & $6(16)$ & \\
\hline Alive & $15(16)$ & $17(37)$ & $7(24)$ & $8(22)$ & \\
\hline Not available & $8(9)$ & $6(13)$ & $5(17)$ & $2(5)$ & \\
\hline Stage & & & & & 0.005 \\
\hline 1 & $51(54)$ & $32(70)$ & $22(76)$ & $31(84)$ & \\
\hline$\|$ & $43(46)$ & $13(28)$ & $7(24)$ & $6(16)$ & \\
\hline Tumor grade EC & & & & & NA \\
\hline FIGO grade I & NA & $11(24)$ & NA & NA & \\
\hline FIGO grade II & NA & $27(59)$ & NA & NA & \\
\hline FIGO grade III & NA & $8(17)$ & NA & NA & \\
\hline Dualistic model & & & & & 0.643 \\
\hline Type I & $0(0)$ & $46(100)$ & $29(100)$ & $37(100)$ & \\
\hline Type ॥ & $94(100)$ & $0(0)$ & $0(0)$ & $0(0)$ & \\
\hline CA125 & & & & & 0.106 \\
\hline$<35$ & $17(18)$ & $13(28)$ & $10(35)$ & $14(38)$ & \\
\hline $35-65$ & $17(18)$ & $7(15)$ & $8(28)$ & $8(22)$ & \\
\hline$>65$ & $60(64)$ & $25(54)$ & $11(38)$ & $15(41)$ & \\
\hline Not available & $0(0)$ & $1(2)$ & $0(0)$ & $0(0)$ & \\
\hline Ploidy & & & & & 0.168 \\
\hline Near diploid & $22(23)$ & $17(37)$ & $7(24)$ & $5(14)$ & \\
\hline Aneuploid & $69(73)$ & $26(57)$ & $19(66)$ & $30(81)$ & \\
\hline Not available & $3(3)$ & $3(7)$ & $3(10)$ & $2(5)$ & \\
\hline Chemotherapy & & & & & 0.212 \\
\hline Yes & $91(97)$ & $42(91)$ & 27 (93) & $37(100)$ & \\
\hline No & $0(0)$ & $0(0)$ & $0(0)$ & $0(0)$ & \\
\hline Not available & $3(3)$ & $4(9)$ & $2(7)$ & $0(0)$ & \\
\hline
\end{tabular}

histotypes (HGSC, EC, MC, CCC) and International Federation of Gynecology and Obstetrics (FIGO) stages. If an antibody dilution of 1:25 resulted in weak or no staining, no further dilutions were tested. One sample in the optimization panel was chosen as positive control for each immunohistochemical experiment. Immunostaining was performed for each protein using the optimized antibody dilutions (Table 2). The sections were immunostained on a Dako Autostainer Plus (Agilent Technologies) using Dako EnVision FLEX visualization systems. More specifically, deparaffinization and antigen retrieval were performed using EnVision FLEX high $\mathrm{pH}$ target retrieval solution ( $\mathrm{pH} 9)$, the sections were stained using liquid DAB (3,3'-diaminobenzidine) 2-component system, and subsequently counterstained with EnVision FLEX hematoxylin (link). After immunostaining, the sections were rinsed with deionized water, dehydrated in an ethanol series comprised of 70,95 and $100 \%$ ethanol, cleared in xylene and mounted.

\section{Determination of immunoreactive score}

Microscopic evaluation of immunostained tissue sections was performed by two pathologists (AK and EWR; blinded to the survival data) based on the percentages and staining intensity (weak, moderate, strong) in tumor cells, as well as the staining intensity in peritumoral stromal and normal cells, to distinguish protein staining intensities in different cell types within the tumor. An immunoreactive score (Hscore) was calculated for each tumor specimen based on the percentage and intensity of positively stained tumor cells, where $0=$ negative, $1=$ weak positive, $2=$ moderate positive and $3=$ strong positive staining. The $\mathrm{H}$-score values ranged between 0 and 300 , where $\mathrm{H}$-score equaled $(1 \times \mathrm{x} \% 1)+(2 \times \mathrm{x} \%)+(3 \times \mathrm{x} \% 3)$ [21]. The $\mathrm{H}$-score was used to correlate the protein expression levels to OS and DSS. An H-score cutoff stratifying the tumor specimens in positive and negative protein expression was determined for each protein using Kaplan-Meier plots in X-tile Software (v. 3.6.1) [22].

\section{Statistical analysis}

Statistical analyses were performed using $P$ values $<0.05$ (two-sided) in R/Bioconductor v. 3.5.1. Univariable and multivariable Cox proportional hazard models were calculated for COL3A1, GPR158 and PITHD1 expression in relation to OS (defined as the time from initial diagnosis to death from any cause) and DSS (defined as the time from initial diagnosis to ovarian cancer-related death) using the 95 RNA sequenced samples for COL3A1, MC samples for GPR158, and CCC samples for PITHD1. Kaplan-Meier curves were generated and tested with log rank tests using survival time and dichotomized $\mathrm{H}$-score for positive immunostaining (survival v. $2.40-1$, survminer v. 0.4 .3$)[23,24]$. The relationship between clinicopathological parameters and positive/negative protein expression were calculated using two-tailed Fisher's exact test (tableone v. 0.9.3) [25]. Multivariable Cox proportional hazard models were used to assess the 
Table 2 Statistical characteristics for the study genes and selected antibodies and corresponding optimized antibody dilution factors for $\mathrm{IHC}$ experiment. Twenty-seven genes are listed in view of their respective HR, 95\% Cl, $P$ value, C-index and log2 ratio. MTUS1 and COL3A1 were selected in view of the findings in our previous work. An optimized dilution factor for IHC analysis could be determined for 12 of the 29 proteins. All antibodies with determined optimized dilution were polyclonal antibodies except the antibody for PTEN which was monoclonal

\begin{tabular}{|c|c|c|c|c|c|c|c|c|c|c|}
\hline Gene symbol & Histotype & Survival & $\mathrm{HR}$ & $95 \% \mathrm{Cl}$ & $P$ Value & C-index & Log2 ratio & Antibody & Company & $\overline{\text { Optimized dilution }}$ \\
\hline ARHGAP21 & EC & OS & 1.00 & $1.00-1.003$ & 0.0011 & 0.80 & 1.53 & 22,183-1-AP & Nordic Biosite & $1: 50$ \\
\hline$A R M C 3$ & HGSC & OS & 0.80 & $0.70-0.92$ & 0.0013 & 0.64 & -3.83 & HPA037823 & Sigma-Aldrich & - \\
\hline C7 & $\mathrm{EC}$ & OS & 1.32 & $1.03-1.69$ & 0.0310 & 0.72 & 5.55 & HPA001465 & Sigma-Aldrich & - \\
\hline $\mathrm{CDH} 18$ & HGSC & OS & 1.18 & $1.06-1.32$ & 0.0020 & 0.64 & 5.67 & HPA014416 & Sigma-Aldrich & - \\
\hline CES3 & EC & OS & 0.66 & $0.49-0.90$ & 0.0088 & 0.74 & -4.17 & HPA041008 & Sigma-Aldrich & $1: 500$ \\
\hline COL11A1 & $\mathrm{CCC}$ & OS & 1.49 & $1.13-1.96$ & 0.0042 & 0.77 & 4.32 & ab64883 & Abcam & - \\
\hline EHD3 & $\mathrm{CCC}$ & DSS & 11.67 & $2.23-59.35$ & 0.0031 & 0.95 & 2.50 & HPA049890 & Sigma-Aldrich & - \\
\hline FRMPD2 & EC & OS & 0.76 & $0.60-0.96$ & 0.0228 & 0.71 & -5.34 & HPA045059 & Sigma-Aldrich & - \\
\hline GABRP & $\mathrm{CCC}$ & OS & 1.34 & $1.03-1.73$ & 0.0275 & 0.71 & 5.05 & PA5-46830 & Thermo Fisher & - \\
\hline GID4 & $\mathrm{EC}$ & OS & 1.04 & $1.02-1.07$ & 0.0013 & 0.79 & 0.75 & HPA044348 & Sigma-Aldrich & $1: 150$ \\
\hline GPR158 & $M C$ & OS & 1.44 & $1.02-2.04$ & 0.0380 & 0.77 & 4.93 & HPA013185 & Sigma-Aldrich & $1: 25$ \\
\hline GRM5 & EC & OS & 0.56 & $0.35-0.91$ & 0.0179 & 0.73 & -4.17 & ab76316 & Abcam & - \\
\hline$|G H G|$ & $\mathrm{CCC}$ & DSS & 1.20 & $1.04-1.39$ & 0.0128 & 0.72 & 7.40 & SAB1401207 & Sigma-Aldrich & $1: 25$ \\
\hline JCHAIN & $\mathrm{CCC}$ & OS & 1.23 & $1.04-1.46$ & 0.0133 & 0.73 & 6.34 & HPA044132 & Sigma-Aldrich & - \\
\hline KIF26B & EC & DSS & 0.46 & $0.23-0.91$ & 0.0248 & 0.91 & -3.68 & HPA027709 & Sigma-Aldrich & - \\
\hline MAP7D2 & $\mathrm{MC}$ & OS & 1.86 & $1.09-3.16$ & 0.0218 & 0.83 & 4.06 & HPA051508 & Sigma-Aldrich & - \\
\hline MTRNRZLI & HGSC & OS & 1.23 & $1.08-1.40$ & 0.0017 & 0.64 & 5.68 & HPA059729 & Sigma-Aldrich & - \\
\hline MUC15 & EC & OS & 0.66 & $0.50-0.87$ & 0.0032 & 0.79 & -5.33 & HPA026110 & Sigma-Aldrich & - \\
\hline PITHD1 & $\mathrm{CCC}$ & DSS & 146.53 & $6.19-3470$ & 0.0020 & 0.89 & 0.57 & PAB20914 & Abnova & $1: 50$ \\
\hline PTEN & $\mathrm{EC}$ & DSS & 404.90 & $1.23-133,809$ & 0.0425 & 0.96 & 0.99 & ab109454 & Abcam & $1: 100$ \\
\hline RTKN2 & $M C$ & OS & 7.87 & $1.48-41.96$ & 0.0157 & 0.89 & 1.66 & 17,458-1-AP & Nordic Biosite & $1: 25$ \\
\hline SLC9A4 & $M C$ & OS & 0.65 & $0.44-0.96$ & 0.0292 & 0.76 & -4.65 & HPA036096 & Sigma-Aldrich & - \\
\hline SMYD2 & $\mathrm{CCC}$ & DSS & 56.79 & $3.92-822.37$ & 0.0031 & 0.89 & 0.91 & PA5-51339 & Thermo Fisher & - \\
\hline TRIM71 & HGSC & DSS & 0.78 & $0.66-0.92$ & 0.0036 & 0.67 & -4.30 & HPA038141 & Sigma-Aldrich & - \\
\hline TRIO & $\mathrm{CCC}$ & DSS & 19.72 & $2.47-157.47$ & 0.0049 & 0.93 & 1.27 & HPA008157 & Sigma-Aldrich & $1: 25$ \\
\hline$\pi K$ & $M C$ & OS & 5.76 & $1.52-21.83$ & 0.0100 & 0.88 & 1.98 & PAB3320 & Abnova & $1: 25$ \\
\hline VNN1 & $\mathrm{CCC}$ & OS & 1.36 & $1.05-1.75$ & 0.0184 & 0.71 & 4.48 & HPA064145 & Sigma-Aldrich & - \\
\hline COL3A1 & 95 RNA-seq samples & OS & - & - & - & - & - & HPA007583 & Sigma-Aldrich & $1: 50$ \\
\hline MTUS1 & 95 RNA-seq samples & OS & - & - & - & - & - & ab198176 & Abcam & $1: 40$ \\
\hline
\end{tabular}

predictive strength (C-index) of COL3A1, GPR158 and PITHD1 when adjusted by established clinical parameters (age, stage, CA125, ploidy and/or histotype). Box plots were generated to compare RNA-protein expression and differences in $\mathrm{H}$-score between the histotype and survival groups using ggplot2 (v. 3.1.0) and Kruskal-Wallis test [26]. For external validation, the Kaplan-Meier (KM) plotter online tool (http://kmplot.com/analysis/) for ovarian cancer $(n=1657)$ was used to determine the clinical relevance of gene expression for the study genes in relation to OS [27]. The REMARK reporting recommendations for prognostic biomarkers were applied to this study (Additional file 5: Table S1) [28].

\section{Results}

\section{Selection of study genes with prognostic value}

To identify genes with prognostic value for each histotype, Cox regression models were used to generate genetic signatures with raw RNA-seq read counts in relation to survival data (OS, DSS). In total, 2223 and 2261 genes with $P$ values $<0.05$ were identified in HGSC for OS and DSS. For EC, 1440 and 522 genes, and 3557 and 1827 genes for CCC were associated with OS and DSS. For MC, 970 genes were significantly associated with OS. Nine promising prognostic genes were selected among the top 10 genes with the highest $\mathrm{C}$-index (Table 2). A further selection of genes was additionally based on $\log 2$ 
ratio. In HGSC, 670 and 1278 genes with $P$ values $<0.05$, C-index $>0.6$ and were identified for OS and DSS. With regard to EC, 512 (OS) and 521 genes (DSS), 1080 (OS) and 1501 genes (DSS) for CCC, and 885 genes (OS) for $\mathrm{MC}$ were associated with prognosis $(P$ values $<0.05$, $C$-indices $>0.7)$. Eighteen promising prognostic genes were selected among the top 20 genes with the highest $\log 2$ ratio $(\log 2$ ratio $>|3.8|$ for HGSC, $\log 2$ ratio $>|3.5|$ for EC, MC, CCC) (Table 2). The selection of biomarkers to be tested with IHC was also based on high gene expression levels and a variation in gene expression depending on histotype-specific survival rates. The previously identified genes, MTUS1 and COL3A1, wherein carriers of MTUS1 frameshift insertion associated with a significant difference in gene expression and OS, and COL3A1 gene expression correlated with tumor aggressiveness, were also included in the selection [13].

\section{IHC analysis identified histotype-specific aberrant protein expression patterns}

Optimized antibody dilutions could be determined for 12 (ARHGAP21, CES3, GID4, GPR158, IGHG1, PITHD1, PTEN, RTKN2, TRIO, TTK, COL3A1, and MTUS1) of the 29 proteins using the optimization panel (Table 2). Seventeen antibodies had either no or too weak staining at 1:25 and were therefore not studied further. IHC was used to evaluate COL3A1 and MTUS1 protein expression levels in the 95 RNA sequenced samples, whereas the remaining 10 proteins were examined in respective histotypes (Table 2). ARHGAP21 and IGHG1 were strongly expressed in both stromal and tumor cells, and therefore excluded from further study. Positive staining was interpreted as $\mathrm{H}$-score $>20$ for COL3A1, $>0$ for GPR158, and $>60$ for PITHD1, while no significant $\mathrm{H}$-score cutoff could be determined for CES3, GID4, MTUS1, PTEN, TRIO and TTK. However borderline significance was found for RTKN2 protein expression in $\mathrm{MC}$ samples in relation to OS $(P$ value $=$ 0.054, HR = 3.94 (0.89-17.50), H-score cutoff > 90).

Generally, the immunostaining patterns for COL3A1, GPR158 and PITHD1 were homogeneous with the exception of two samples (the staining intensity for one COL3A1 sample and one GPR158 sample were evaluated as weak-moderate staining). COL3A1 protein expression varied in the 95 RNA sequenced ovarian carcinoma samples. The IHC results showed that the COL3A1 protein was mainly localized to the cytoplasm of tumor cells (Fig. 1a) and the vast majority of samples were COL3A1-positive $(n=87,92 \%)$. Positive COL3A1 staining was detected in all histotypes, whereas negative staining was not detected in MC. GPR158 immunostaining was evaluated in the MC histotype and was found to show GPR158-positivity in $17 / 29$ patients (59\%). The GPR158 protein was mainly localized to the cytoplasm of tumor cells, but occasional staining was also found in tumor cell nuclei. Finally, PITHD1-positivity was found in the vast majority of the CCC samples $(n=34,92 \%)$, with positive immunostaining primarily observed in tumor cell nuclei (Fig. 1a). In addition, slight to moderate expression of PITHD1 were also seen in stromal cells. No association was found between COL3A1, GPR158 or PITHD1 protein expression and clinicopathological characteristics (Additional file 6: Table S2). COL3A1 expression was predominantly observed as intermediate staining, GPR158 as weak staining and PITHD1 as strong staining (Additional file 1: Figure S1). The $\mathrm{H}$-score for COL3A1 expression varied depending on histotype, with low COL3A1 expression levels in CCC samples $(P$ value $=0.0015$, Additional file 2: Figure S2a), particularly samples in the $5-10$ year survival group $(P$ value $=0.001$, Additional file 2: Figure S2b $)$.

The $\mathrm{H}$-score data was further compared with the raw RNA-seq read counts, by converting both datasets to $\log 2$ values. For GPR158 and PITHD1, two analyses were performed with 1) comparing RNA-seq read counts for the 95 RNA sequenced samples with H-score values for the patient cohort, and 2) comparing RNAseq read counts with corresponding $\mathrm{H}$-score values within the 95 RNA sequenced samples. For both analyses, no difference was found between the RNA and protein expression patterns for GPR158 and PITHD1 (first analysis: GPR158 $P$ value $=0.54$, PITHD1 $P$ value $=$ 0.11 , second analysis: GPR158 $P$ value $=0.66$, PITHD $1 P$ value $=0.68$. COL3A1 protein expression was lower than COL3A1 RNA expression, which was also seen when stratified by histotype (Fig. 1b and c).

\section{Kaplan-Meier analysis reveals the prognostic value of COL3A1, GPR158 and PITHD1 protein expression}

Kaplan-Meier curves and log-rank tests were used to estimate patient survival in relation to COL3A1, GPR158 and PITHD1 protein expression levels. The Kaplan-Meier curves were dichotomized according to the $\mathrm{H}$-score cutoff for positive immunostaining. Kaplan-Meier analysis revealed an association between COL3A1, GPR158 and PITHD1 protein expression with OS and/or DSS (Fig. 2). More specifically, COL3A1 expression was associated with shorter OS rates $(P$ value $=0.026, \mathrm{HR}=2.99(95 \% \mathrm{CI}$ 1.089-8.19)) in epithelial ovarian carcinoma patients. A significant association was not found for COL3A1 expression and DSS. Furthermore, GPR158 expression was associated with shorter OS and DSS $(P$ value $=0.00043$, $\mathrm{HR}=6.13(95 \%$ CI $1.98-18.98) ; P$ value $=0.029)$ in $\mathrm{MC}$ patients. Lastly, PITHD1 expression was associated with longer OS and DSS $(P$ value $=0.012, \mathrm{HR}=0.22(95 \% \mathrm{CI}$ $0.058-0.80) ; P$ value $=0.003, \mathrm{HR}=0.17$ (95\% CI $0.043-$ 0.64)) in CCC patients. 


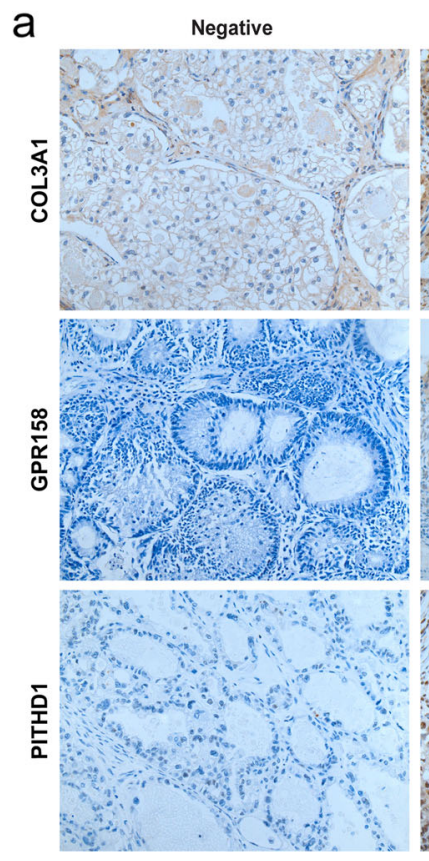

b

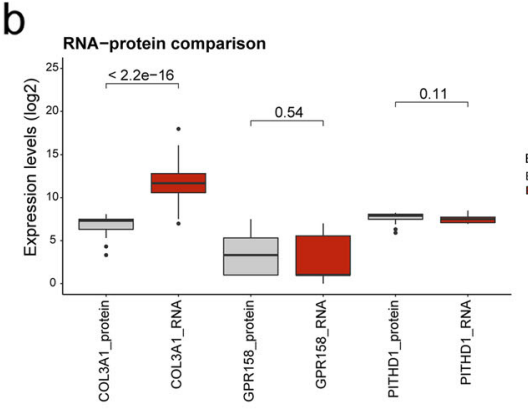

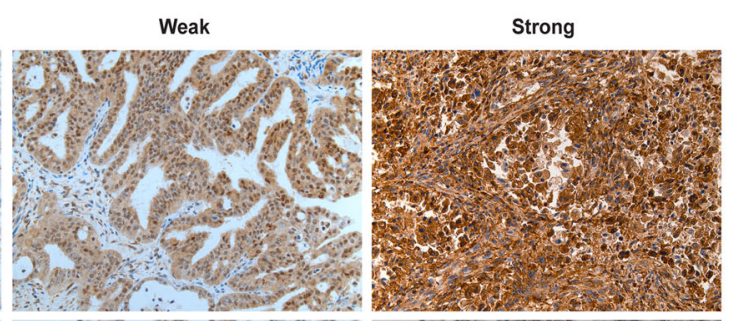
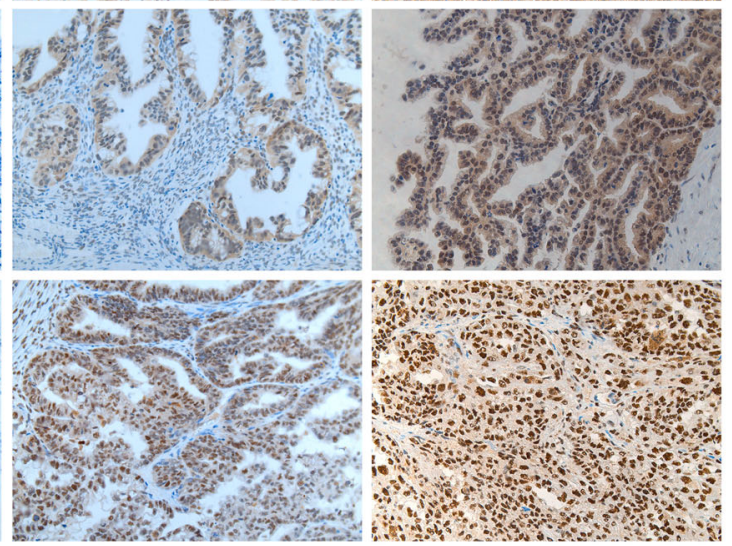

C

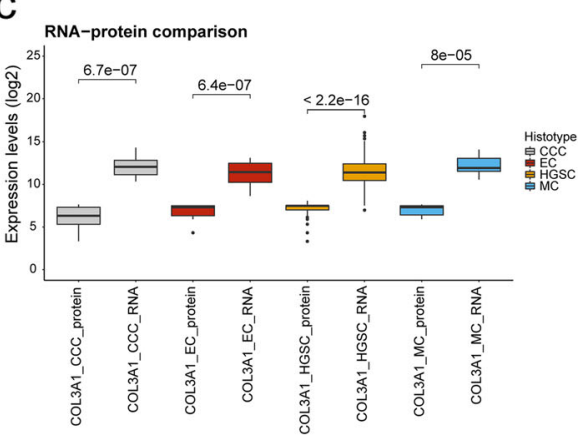

Fig. 1 COL3A1, GPR158, PITHD1 protein expression in ovarian tumor cells and comparison between RNA and protein expression. a) shows representative IHC staining intensities (negative-strong) in hot spots for COL3A1, GPR158 and PITHD1 (200 x magnification). COL3A1 was shown to be negative, weak and strong in the CCC, EC and HGSC histotypes. Box plots illustrating the comparison between the distributions of log2 values of raw RNA-seq read counts and H-score for COL3A1, GPR158 and PITHD1, with b) all H-score values are taken into account. Similar RNA and protein expression is shown for GPR158 and PITHD1 (GPR158 $P$ value $=0.54$, PITHD1 $P$ value $=0.11$ ). For COL3A1, higher RNA expression in comparison with protein expression was revealed. This was also seen in c) when stratified by histotype

\section{Predictive performance is improved when combining} COL3A1, GPR158 and PITHD1 expression with established clinicopathological parameters

Univariate and multivariable survival analyses for OS and DSS were performed to evaluate whether COL3A1, GPR158 and PITHD1 expression could improve outcome prediction. When adjusted for established clinical parameters (age, stage, CA125, ploidy and/or histotype), COL3A1 expression improved outcome prediction for OS $(\mathrm{HR}=3.2, P$ value $=0.032)$, whereas the predictive performance of GPR158 and PITHD1 expression was improved for OS $(\mathrm{HR}=23.83, P$ value $=0.00011)$, and OS and DSS $(\mathrm{HR}=0.082, P$ value $=0.0013 ; \mathrm{HR}=0.047, P$ value $=0.00036) \quad($ Table 3$)$. However, the improvement in outcome prediction was most prominent for GPR158 and PITHD1. The predictive model for GPR158 and OS showed the highest increase in $\mathrm{C}$-index from 0.627 for established parameters to 0.795 in combination with GPR158 protein status (Additional file 3: Figure S3).

$\mathrm{KM}$ plotter confirms the prognostic value of $\mathrm{COL} 3 A 1$, GPR158 and PITHD1 gene expression

KM plotter was used to validate the prognostic value of COL3A1, GPR158 and PITHD1 in an external ovarian cancer dataset. The Kaplan-Meier curves were dichotomized according to the median value of expression, wherein patient samples with expression levels above the median were placed in the high expression group and patient samples with expression levels below the median were classified in the low expression group. Fig. 3 shows 


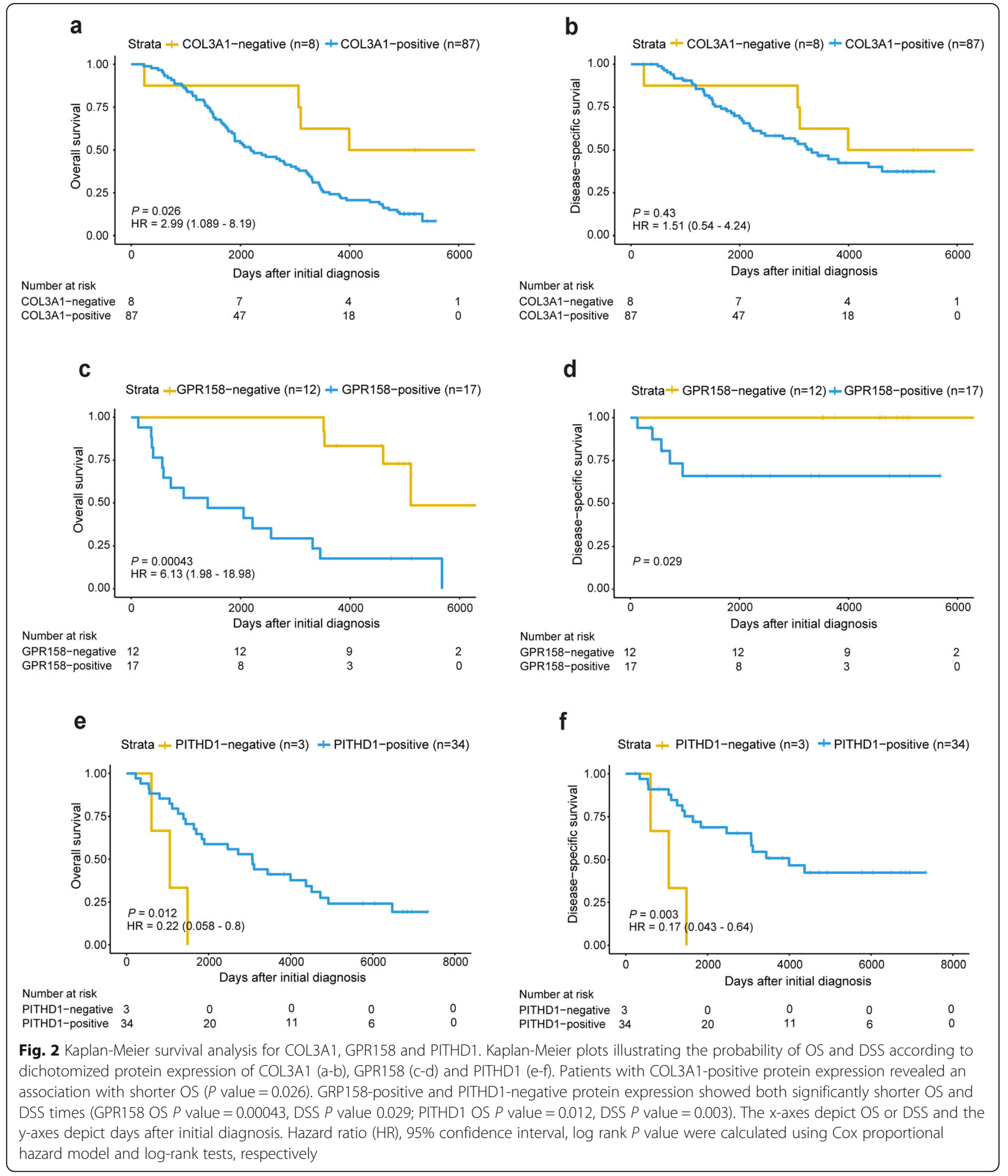

significant Kaplan-Meier plots for COL3A1 (Affymetrix ID: 211161 s at, $n=1656$ patients, $P$ value $=7.7 \mathrm{e}-09$ ), GPR158 (Affymetrix ID: 232195_at, $n=655$ patients, $P$ value $=0.014$ ) and PITHD1 (Affymetrix ID: 223124_s_at, $n=655$ patients, $P$ value $=7.9 \mathrm{e}-06)$. All three genes showed significant differences in gene expression levels between the expression groups. High levels of COL3A1, GPR158 and PITHD1 expression correlated with lower patient survival rates. Furthermore, COL3A1 (Affymetrix ID: $201852 \_x \_$at, $P$ value $=2.1 \mathrm{e}-05 ; \quad$ Affymetrix ID: 
Table 3 Univariable and multivariable survival analysis for COL3A1, GPR158 and PITHD1 expression and overall and disease-specific survival. COL3A1 is adjusted for histotype, age, stage, CA125 and ploidy. GPR158 and PITHD1 are adjusted for age, stage, CA125 and ploidy. Significant values are marked in bold

\begin{tabular}{|c|c|c|c|c|c|c|}
\hline & \multicolumn{3}{|c|}{ Overall survival } & \multicolumn{3}{|c|}{ Disease-specific survival } \\
\hline & HR (95\% Cl) & $P$ value & C-index & HR (95\% Cl) & $P$ value & C-index \\
\hline \multicolumn{7}{|c|}{ Univariable analysis } \\
\hline Histotype & - & 0.55 & 0.541 & - & 0.020 & 0.620 \\
\hline Patient age & 1.018 & 0.038 & 0.568 & 0.99 & 0.48 & 0.530 \\
\hline Stage & 1.11 & 0.67 & 0.512 & 2.0 & 0.016 & 0.583 \\
\hline CA125 & - & 0.25 & 0.550 & - & 0.69 & 0.533 \\
\hline Ploidy & - & 0.06 & 0.561 & - & 0.36 & 0.551 \\
\hline COL3A1 & 2.99 & 0.026 & 0.537 & 1.51 & 0.43 & 0.520 \\
\hline \multicolumn{7}{|c|}{ Multivariable analysis } \\
\hline COL3A1 & 3.20 & 0.032 & 0.654 & 1.40 & 0.56 & 0.704 \\
\hline \multicolumn{7}{|c|}{ Univariable analysis } \\
\hline Patient age & 1.02 & 0.18 & 0.591 & 0.98 & 0.46 & 0.598 \\
\hline Stage & 1.07 & 0.89 & 0.450 & 4.57 & 0.068 & 0.684 \\
\hline CA125 & - & 0.97 & 0.493 & - & 0.45 & 0.642 \\
\hline Ploidy & - & 0.59 & 0.569 & - & 0.23 & 0.689 \\
\hline GPR158 & 6.13 & 0.00043 & 0.690 & - & 0.029 & 0.748 \\
\hline \multicolumn{7}{|c|}{ Multivariable analysis } \\
\hline GPR158 & 23.83 & 0.00011 & 0.795 & - & 1.0 & 0.946 \\
\hline \multicolumn{7}{|c|}{ Univariable analysis } \\
\hline Patient age & 1.021 & 0.22 & 0.569 & 1.014 & 0.48 & 0.548 \\
\hline Stage & 1.04 & 0.94 & 0.505 & 0.73 & 0.68 & 0.522 \\
\hline CA125 & - & 0.57 & 0.558 & - & 0.22 & 0.601 \\
\hline Ploidy & - & 0.23 & 0.542 & - & 0.12 & 0.560 \\
\hline PITHD1 & 0.22 & 0.012 & 0.558 & 0.17 & 0.003 & 0.574 \\
\hline \multicolumn{7}{|c|}{ Multivariable analysis } \\
\hline PITHD1 & 0.082 & 0.0013 & 0.694 & 0.047 & 0.00036 & 0.733 \\
\hline
\end{tabular}

215076_s_at, $P$ value $=3.8 \mathrm{e}-06$ ) and PITHD1 (Affymetrix ID: $223123 \_s \_a t, ~ P$ value $\left.=0.043\right)$ were also significantly associated with OS using the KM plotter (Additional file 4: Figure S4). A similar correlation between GPR158 and PITHD1 expression with lower OS were also obtained when tested in relation to OS for EC patients (GPR158: Affymetrix ID: 232195 at, $n=30$ patients, $P$ value $=0.063$; PITHD1: Affymetrix ID: $223124, n=30$ patients, $P$ value $=$ $0.0004)$. Moreover, $21 / 26$ study genes were significantly associated with patient survival for at least one Affymetrix ID probe (Additional file 7: Table S3). The FRMPD2, SLC9A4 and TRIM71 genes are not included on the GeneChip $^{\text {тм }}$ Human Genome U133A 2.0 Array and could therefore not be assessed with the KM plotter.

\section{Discussion}

In the current study, the prognostic role of 29 genes was assessed in early-stage ovarian carcinomas using IHC.
The patient samples used for biomarker validation represented a large cohort of early-stage ovarian carcinoma specimens $(n=206)$ distributed across four histotypes $(\operatorname{HGSC}(n=94)$, EC $(n=46), \mathrm{MC}(n=29)$ and CCC $(n=$ $37)$ ). The MTUS1 and COL3A1 genes were chosen from our previous study [13] and the remaining 27 genes showed a promising correlation between RNA-seq expression and survival rates (OS and DSS) in different histotypes (HGSC, EC, MC and CCC). Unfortunately, antibodies for all of the 29 proteins could not be optimized, in part, due to potential discrepancies between transcript and protein expression levels, too low gene expression or unsuitable antibodies. RNA expression is a suitable indicator for protein level, but it does not always directly correlate with protein expression due to e.g. post transcriptional modification, translational and protein degradation regulation $[29,30]$. It has been reported that intratumoral heterogeneity has limited impact on gene 


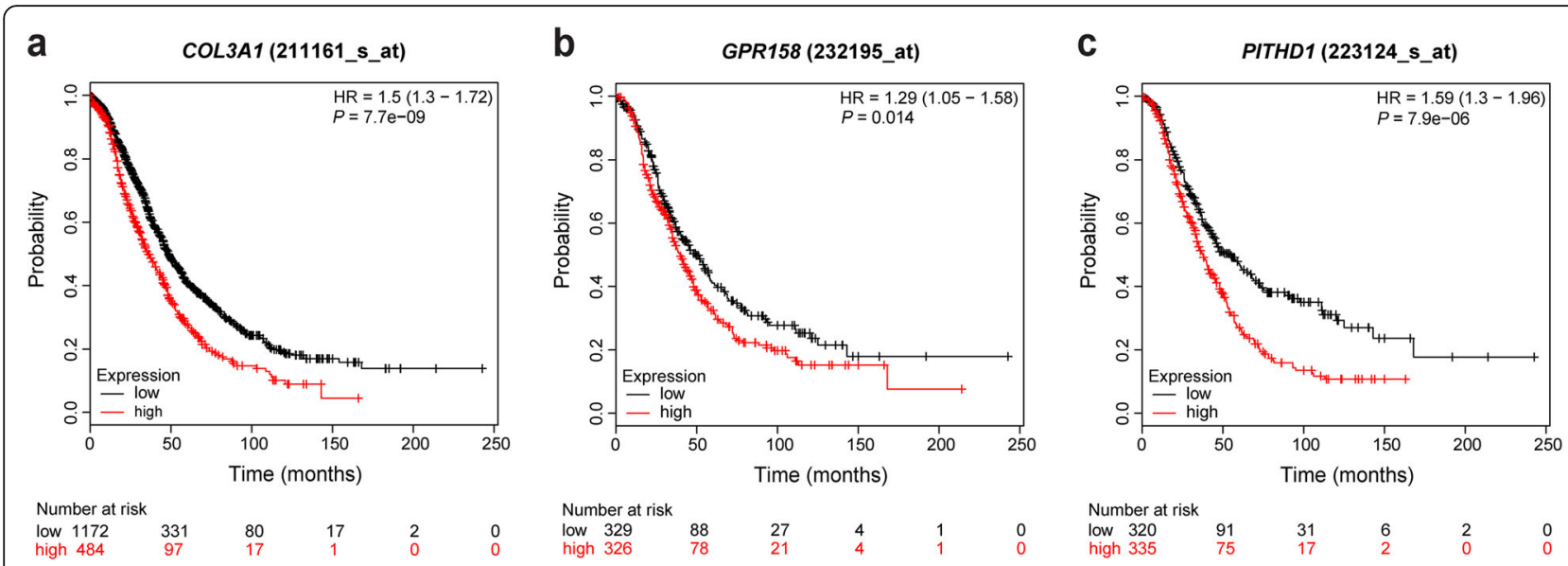

Fig. 3 Validation of prognostic value of COL3A1, GPR158 and PITHD1 using KM plotter. Kaplan-Meier plots showing overall survival in HGSC and EC for a) COL3A1 ( $n=1656$ patients), b) GPR158 ( $n=655$ patients) and b) PITHD1 ( $n=655$ patients). Red: patient samples with expression levels above the median, black: patient samples with expression levels below the median. $P$ values less than 0.05 were considered significant. Numberat-risk is indicated below the main plot. Hazard ratio (HR), 95\% confidence interval, log rank $P$ value were calculated using Cox proportional hazard model and log-rank tests, respectively

expression profiling and can be easily detected using IHC $[31,32]$.

Survival analysis revealed that the RNA expression levels for the COL3A1, GPR158 and PITHD1 genes were significantly correlated with that shown on the protein expression levels, proposing them as prognostic factors for ovarian carcinoma (COL3A1) and in MC (GPR158) and CCC histotypes (PITHD1). COL3A1 is part of the collagen family which may affect the tumor microenvironment to promote tumor progression by regulating the extracellular matrix via collagen degradation and redeposition [33, 34]. GPR158 is an orphan class C member belonging to the G Protein coupled receptor (GPCR) superfamily of cell-surface signaling proteins which have been shown to exhibit aberrant expression in multiple cancers such as colon, breast, prostate and ovarian cancer, thereby contributing to e.g. tumor progression and metastasis [35, 36]. Furthermore, GPCRs are involved in many physiological and disease processes, making them important therapeutic targets [37]. This is reflected by GPCRs being the target of about $34 \%$ of all FDA-approved drugs [38]. Little is known about PITHD1. A recent study reported that PITHD1 is downregulated in leukemia and may regulate RUNX1 expression that promotes megakaryocyte differentiation, and activates the internal ribosomal entry site [39].

The IHC results showed that COL3A1 protein staining was mainly localized in the cytoplasm of tumor cells, but at varying intensity in the 95 RNA sequenced samples. Kruskal-Wallis analysis of variance also indicated that COL3A1 expression was dependent on histotype with lower expression in CCC patients. Previously, a variation in COL3A1 protein staining has been reported in e.g. epithelial tumor cells, in the cytoplasm but also in the nucleus of colorectal carcinoma, using 13,548-1-AP antibody from Proteintech [40]. As reported here, GPR158 was mainly localized in the cytoplasm of tumor cells, with occasional staining in the nuclei of tumor cells. The HPA database reports cytoplasmic, membranous and nuclear IHC staining for GPR158 in various tissues. Moreover, PITHD1 was herein primarily observed in tumor nuclei. Apart from a recent report where PITHD1 was detected in the cytoplasm of leukemic cells, little information is known about PITHD1 protein staining [39]. Similar expression levels for GPR158 and PITHD1 were observed on the RNA and protein levels. For COL3A1, higher RNA expression was demonstrated in comparison with protein expression in the whole cohort and in relation to each histotype. The lower COL3A1 protein expression may be explained by processes such as post transcriptional modification, translational and protein degradation regulation [29, 30].

The Kaplan-Meier analysis of dichotomized COL3A1 protein expression revealed an association with shorter OS, which is consistent with COL3A1 RNA expression shown in our previous work [13]. A recent report identified COL3A1 in a 7-gene signature related to stage in SC patients contributing to extracellular matrix interactions and mitosis [41], supporting our findings that COL3A1 may be a promising prognostic factor for ovarian tumor progression. Moreover, COL3A1, COL5A2 and COL1A2 expression are associated with drug-resistance in ovarian cancer [42]. However, the exact role of COL3A1 in ovarian tumorigenesis is yet to be revealed. COL3A1 has also been reported in other cancer types such as breast and colorectal cancers, with an association between COL3A1 and P4HA2 with poor prognosis in breast cancer, and correlation between elevated epithelial COL3A1 protein 
expression and unfavorable outcome in colorectal cancer $[40,43]$. It has further been shown that Col3 suppresses metastatic processes of triple-negative breast cancer cells as well as tumor growth and metastasis in mice [44].

Survival analysis of GPR158-positive protein expression in MC patients showed an association with unfavorable OS which is consistent with that found on RNA expression level. Surprisingly, none of the MC ovarian carcinoma patients classified in the GPR158-negative expression group died of ovarian carcinoma. GPR158 has previously been shown to be involved in prostate cancer growth and progression, wherein up-regulation of GPR158 in Pten homozygous knock-out mice could contribute to tumor invasion [45]. Moreover, GPR158 has been shown to promote glioma stem cell differentiation and apoptosis $[45,46]$. To our knowledge, no association has previously been shown for GPR158 expression and ovarian cancer. However, other genes of the GPCR family, such as GRP137 has been shown to be highly expressed in ovarian cancer tissue and gene knockdown resulted in a decrease in cell proliferation rates and inhibition of cell migration capabilities [47]. Furthermore, nuclear expression of GPR30 has been reported to be a negative prognostic factor for OS in epithelial ovarian cancer [48]. Expression of GPR56 in ovarian serous carcinoma was shown to be associated with advanced FIGO stage and to promote progression and invasion of epithelial ovarian cancer [49]. Therefore, GPR158 may present a novel prognostic factor for $\mathrm{MC}$ patients and may constitute a promising novel therapeutic target.

CCC patients with negative PITHD1 protein expression were associated with both significantly shorter OS and DSS. These results are not in line with that found on the RNA level, where elevated PITHD1 expression was correlated with an unfavorable outcome. This contradiction may be due to the different techniques used, e.g. RNA-seq takes into account the transcriptomic expression of all cell types in the tumor specimen (e.g. tumor and stromal cells) whereas immunohistochemistry was scored solely using protein expression patterns in tumor cells. Hence, the discrepancy between PITHD1 RNA and protein levels may be due to the contribution of PITHD1 expression in stromal cells. To our knowledge, no association between PITHD1 expression and ovarian carcinoma has previously been shown and may therefore present a novel prognostic predictor for tumor progression in CCC patients.

Furthermore, combined predictive models containing protein expression status (COL3A1, GPR158 or PITHD1) together with established clinical parameters improved outcome prediction (increased C-index values) compared with models containing established clinical parameters alone, supporting the importance of these biomarkers. The results were further validated in an external cohort using the KM plotter database. RNA expression for all three genes were validated wherein an elevated RNA expression for COL3A1, GPR158 and PITHD1 correlated with unfavorable outcome. However, it should be noted that the majority of the samples in KM plotter datasets were advanced stage (III + IV) in the HGSC or EC histotypes. Unfortunately, there are currently no public databases comprising gene expression data for $\mathrm{CCC}$ or MC patients.

\section{Conclusions}

In summary, we have validated three promising prognostic biomarkers on the protein level in ovarian carcinoma. COL3A1 may play an oncogenic role in epithelial ovarian carcinoma (HGSC, EC, MC, CCC), GPR158 in MC and PITHD1 in CCC, wherein COL3A1 and GPR158 protein expression act as predictors of unfavorable prognosis, whereas PITHD1 protein expression is associated with a favorable prognosis. Our results are interesting in terms of not only prognosis but also tumor progression. The knowledge from this study may in the future ideally be used to assist physicians in prognostication of ovarian carcinoma at the time of diagnosis. Further investigation using e.g. larger patient cohorts, and in vitro and in vivo models are needed to further validate the clinical and biological significance of these biomarkers in ovarian carcinoma histotypes.

\section{Additional files}

Additional file 1: Figure S1. Variation of protein staining intensity in ovarian carcinoma. Pie charts representing the proportion of samples with weak, intermediate or strong staining intensities for each protein. Staining intensities of weak to strong are colored light blue, blue and dark blue. Eight of the nine tumor samples with strong COL3A1 intensity were of HGSC histotype. (TIF $848 \mathrm{~kb}$ )

Additional file 2: Figure S2. Variation in protein expression with regard to histotype and OS. COL3A1 protein expression differed depending on histotype (Additional file 2: Figure S2a) as well as histotype within the 5-10 year survival group (Additional file 2: Figure S2b. The $x$-axes depict COL3A1 H-score and the $\mathrm{y}$-axes depict ovarian carcinoma histotype and survival time, wherein the patients have been stratified into four survival groups 0-2 years, 2-5 years, 5-10 years and > 10 years. (TIF 991 kb)

Additional file 3: Figure S3. Multivariable survival analysis for OS and DSS. The addition of the protein expression status resulted in improved outcome prediction for COL3A1 (a, b), GPR158 (c, d), PITHD1 (e, f). COL3A1 survival analysis was adjusted for histotype, age, stage, CA125, ploidy, and GPR158 and PITHD1 were adjusted for age, stage, CA125, ploidy. The x-axes depict C-index for OS or DSS and the $y$-axes depict survival time in days. C-index values for each outcome prediction curve are shown in parentheses. (TIF $2852 \mathrm{~kb}$ )

Additional file 4: Figure S4. Additional Affymetrix probes for validating COL3A1 and PITHD1 prognostic value using KM plotter. Kaplan-Meier plots showing overall survival in HGSC and EC for a-b) COL3A1 $(n=1656$ patients), and c) PITHD1 ( $n=655$ patients). Red: patient samples with expression levels above the median, black: patient samples with expression levels below the median. $P$ values less than 0.05 were considered significant Number-at-risk is indicated below the main plot. Hazard ratio (HR), 95\% confidence interval, log rank $P$ were calculated using Cox proportional hazard model and log-rank tests. (TIF $1050 \mathrm{~kb}$ )

Additional file 5: Table S1. Reporting recommendations for tumor marker prognostic 642 studies (REMARK) guidelines. (DOCX 22 kb) 
Additional file 6: Table S2. Distribution of clinicopathological characteristics 645 in relation to COL3A1, GPR158 and PITHD1 protein expression. (DOCX $22 \mathrm{~kb}$ )

Additional file 7: Table S3. KM plotter probe ID and $P$ values for the study 647 genes. Twenty-six of the 29 study genes could be evaluated using KM plotter. The FRMPD2, SLC9A4 and TRIM71 genes could not be assessed since they are not included on the GeneChip ${ }^{T M}$ Human Genome U133A 2.0 Array. Twenty one genes showed significant Kaplan-Meier plots for at least one Affymetrix ID probe (marked in bold). (DOCX $26 \mathrm{~kb}$ )

\section{Abbreviations}

95\% Cl: 95\% Confidence Interval; AQP1: Aquaporin-1; AUC(t): Timedependent Area under the receiver operating characteristics curve; CA 125: Cancer antigen 125; CCC: Clear-cell ovarian carcinoma; Cindex: Concordance index; COL3A1: Collagen type III alpha 1 chain; CT 45: Cancer antigen 45; DSS: Disease-specific survival; EC: Endometrioid ovarian carcinoma; FDR: False discovery rate; FFPE: Full-face formalin-fixed paraffin-embedded; FIGO: International Federation of Gynecology and Obstetrics; GEO: Gene Expression Omnibus; GPR158: G protein-coupled receptor 158; HE4: Human epididymis protein 4; HGSC: High-grade serous ovarian carcinoma; HPA: Human Protein Atlas; HR: Hazard ratio; Hscore: Immunoreactive score; IHC: Immunohistochemistry; MC: Mucinous ovarian carcinoma; MIA: Multivariate index assay; OS: Overall survival; PITHD1: PITH domain containing 1; RNA-seq: RNA sequencing; SC: Serous ovarian carcinoma; TCGA: The Cancer Genome Atlas

\section{Acknowledgments}

Not applicable.

\section{Author's contributions}

K.H. and P.K. were responsible for overall study concept, design of experiments, and collection of clinical data. T.Z.P. and H.E. performed the IHC analysis. S.N. performed the statistical analysis correlating gene expression data with survival status for study gene selection, and H.E., T.Z.P. and J.B. performed the remaining statistical analyses. A.K. and E.W.R. performed the pathological reclassification of the histotypes and the microscopic evaluation of stained tissue sections. S.D.H. prepared the FFPE sections on IHC microscope slides. K.S. provided technical support relating to ovarian carcinoma. H.E. planned and prepared the $\mathrm{IHC}$ experiments, analyzed the data and wrote the manuscript. All authors reviewed, edited, and approved the final manuscript.

\section{Funding}

This work was supported by grants from the Swedish Cancer Society (CAN 2018/417), King Gustav V Jubilee Clinic Cancer Research Foundation (2017:144), the LUA/ALF-agreement in West of Sweden health care region, Assar Gabrielsson Research Foundation for Clinical Cancer Research (FB 17-08, FB 18-69), and Sahlgrenska University Hospital Research Foundation. The funders had no role in the design of the study, data collection and analysis, interpretation of data or in writing the manuscript.

\section{Availability of data and materials}

Raw RNA-seq read counts for 95 of the 206 ovarian tumors have been deposited in the NCBI Gene Expression Omnibus (http://www.ncbi.nlm.nih.gov/geo/) under accession number GSE101109.

\section{Ethics approval and consent to participate}

The Regional Ethical Review Board (Gothenburg, Sweden) approved the study with reference number 767-14 and a waiver of written consent to use the tumor specimens. The committee that approved the study were Marika Estreen (chair) and Lennart Andrén (scientific secretary). Further, all procedures were performed in accordance with the Declaration of Helsinki.

\section{Consent for publication}

Not applicable.

\section{Competing interests}

The authors declare that they have no competing interests. Author $\mathrm{KH}$ is an Editorial Board member for the journal.

\section{Author details}

'Department of Oncology, Institute of Clinical Sciences, Sahlgrenska Cancer Center, Sahlgrenska Academy at University of Gothenburg, Gothenburg, Sweden. ${ }^{2}$ Department of Clinical Pathology, Sahlgrenska University Hospital, Gothenburg, Sweden. ${ }^{3}$ Department of Orthopaedics, Institute of Clinical Sciences, Sahlgrenska Academy at University of Gothenburg, Gothenburg, Sweden. ${ }^{4}$ Department of Obstetrics and Gynecology, Institute of Clinical Sciences, Sahlgrenska Academy at University of Gothenburg, Gothenburg, Sweden.

Received: 14 April 2019 Accepted: 23 August 2019

Published online: 18 September 2019

References

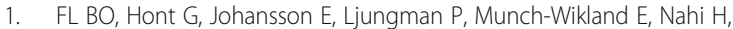
Zedenius J. Cancer i siffror In. Cancerfonden, Socialstyrelsen; 2018.

2. Siegel RL, Miller KD, Jemal A. Cancer statistics, 2018. CA Cancer J Clin. 2018; 68(1):7-30.

3. Lundqvist EÅ. National clinical guidelines for ovarian cancer. Sweden: Regional Cancer Centers; 2015.

4. Torre LA, Trabert B, DeSantis CE, Miller KD, Samimi G, Runowicz CD, Gaudet MM, Jemal A, Siegel RL. Ovarian cancer statistics, 2018. CA Cancer J Clin. 2018;68(4):284-96

5. Soletormos G, Duffy MJ, Othman Abu Hassan S, Verheijen RH, Tholander B, Bast RC Jr, Gaarenstroom KN, Sturgeon CM, Bonfrer JM, Petersen PH, et al. Clinical use of Cancer biomarkers in epithelial ovarian Cancer: updated guidelines from the European group on tumor markers. Int J Gynecol Cancer. 2016;26(1):43-51.

6. Nustad K, Bast RC Jr, Brien TJ, Nilsson O, Seguin P, Suresh MR, Saga T, Nozawa S, Bormer OP, de Bruijn HW, et al. Specificity and affinity of 26 monoclonal antibodies against the CA 125 antigen: first report from the ISOBM TD-1 workshop. International Society for Oncodevelopmental Biology and Medicine. Tumour Biol. 1996;17(4):196-219.

7. Hogdall EV, Christensen L, Kjaer SK, Blaakaer J, Kjaerbye-Thygesen A, Gayther S, Jacobs IJ, Hogdall CK. CA125 expression pattern, prognosis and correlation with serum CA125 in ovarian tumor patients. From the Danish "MALOVA" ovarian Cancer study. Gynecol Oncol. 2007;104(3):508-15.

8. Gadducci A, Cosio S, Carpi A, Nicolini A, Genazzani AR. Serum tumor markers in the management of ovarian, endometrial and cervical cancer. Biomed Pharmacother. 2004;58(1):24-38.

9. Moore RG, McMeekin DS, Brown AK, DiSilvestro P, Miller MC, Allard WJ, Gajewski W, Kurman R, Bast RC Jr, Skates SJ. A novel multiple marker bioassay utilizing HE4 and CA125 for the prediction of ovarian cancer in patients with a pelvic mass. Gynecol Oncol. 2009;112(1):40-6.

10. Coleman RL, Herzog TJ, Chan DW, Munroe DG, Pappas TC, Smith A, Zhang Z, Wolf J. Validation of a second-generation multivariate index assay for malignancy risk of adnexal masses. Am J Obstet Gynecol. 2016;215(1):82.e81-11.

11. Coscia F, Lengyel E, Duraiswamy J, Ashcroft B, Bassani-Sternberg M, Wierer M, Johnson A, Wroblewski K, Montag A, Yamada SD, et al. Multi-level Proteomics Identifies CT45 as a Chemosensitivity Mediator and Immunotherapy Target in Ovarian Cancer. Cell. 2018;175(1):159-170.e116.

12. Sato K, Miyamoto M, Takano M, Furuya K, Tsuda H. Different prognostic implications of Aquaporin-1 and Aquaporin-5 expression among different histological types of ovarian carcinoma. POR: Pathology oncology research; 2018.

13. Engqvist H, Parris TZ, Ronnerman EW, Soderberg EMV, Biermann J, Mateoiu C, Sundfeldt K, Kovacs A, Karlsson P, Helou K. Transcriptomic and genomic profiling of early-stage ovarian carcinomas associated with histotype and overall survival. Oncotarget. 2018;9(80):35162-80.

14. Bodurka DC, Deavers MT, Tian C, Sun CC, Malpica A, Coleman RL, Lu KH, Sood AK, Birrer MJ, Ozols R, et al. Reclassification of serous ovarian carcinoma by a 2-tier system: a gynecologic oncology group study. Cancer. 2012;118(12):3087-94.

15. Kurman RJ. International Agency for Research on Cancer., World Health Organization.: WHO classification of tumours of female reproductive organs. 4th ed. International Agency for Research on Cancer: Lyon; 2014.

16. Prat J. Ovarian carcinomas: five distinct diseases with different origins, genetic alterations, and clinicopathological features. Virchows Arch. 2012; 460(3):237-49.

17. Prat J. Oncology FCoG: FIGO's staging classification for cancer of the ovary, fallopian tube, and peritoneum: abridged republication. J Gynecol Oncol. 2015;26(2):87-9. 
18. Heagerty PJ, Zheng Y. Survival model predictive accuracy and ROC curves. Biometrics. 2005;61(1):92-105.

19. Berglund L, Bjorling E, Oksvold P, Fagerberg L, Asplund A, Szigyarto CA, Persson A, Ottosson J, Wernerus H, Nilsson $\mathrm{P}$, et al. A genecentric human protein atlas for expression profiles based on antibodies. Mol Cell Proteomics. 2008;7(10):2019-27.

20. Uhlen M, Bjorling E, Agaton C, Szigyarto CA, Amini B, Andersen E, Andersson AC, Angelidou P, Asplund A, Asplund C, et al. A human protein atlas for normal and cancer tissues based on antibody proteomics. Mol Cell Proteomics. 2005:4(12):1920-32.

21. McCarty KS Jr, Miller LS, Cox EB, Konrath J, McCarty KS Sr. Estrogen receptor analyses. Correlation of biochemical and immunohistochemical methods using monoclonal antireceptor antibodies. Arch Pathol Lab Med. 1985; 109(8):716-21.

22. Camp RL, Dolled-Filhart M, Rimm DL. X-tile: a new bio-informatics tool for biomarker assessment and outcome-based cut-point optimization. Clin Cancer Res. 2004;10(21):7252-9.

23. Therneau T. A Package for Survival Analysis in S; 2015:R package version. p. 2.40-1.

24. Kassambara AK, M.: Survminer - Drawing Survival Curves using 'ggplot2'. 2019:R package version 0.4.3.

25. Yoshida K: tableone: Create 'Table 1' to Describe Baseline Characteristics. 2019:R package version 0.9.3.

26. Wickham H: ggplot2: Elegant Graphics for Data Analysis. 2016:R package version 3.1.0.

27. Gyorffy B, Lanczky A, Szallasi Z. Implementing an online tool for genome-wide validation of survival-associated biomarkers in ovarian-cancer using microarray data from 1287 patients. Endocr Relat Cancer. 2012;19(2):197-208.

28. Altman DG, McShane LM, Sauerbrei W, Taube SE. Reporting recommendations for tumor marker prognostic studies (REMARK): explanation and elaboration. PLoS Med. 2012;9(5):e1001216.

29. Vogel C, Marcotte EM. Insights into the regulation of protein abundance from proteomic and transcriptomic analyses. Nat Rev Genet. 2012;13(4):227-32.

30. Wang D. Discrepancy between mRNA and protein abundance: insight from information retrieval process in computers. Comput Biol Chem. 2008;32(6):462-8.

31. Karthik GM, Rantalainen M, Stalhammar G, Lovrot J, Ullah I, Alkodsi A, Ma R, Wedlund L, Lindberg J, Frisell J, et al. Intra-tumor heterogeneity in breast cancer has limited impact on transcriptomic-based molecular profiling. BMC Cancer. 2017;17(1):802

32. Jiang W, Dulaimi E, Devarajan K, Parsons T, Wang Q, Liao L, Cho EA, O'Neill R, Solomides C, Peiper SC, et al. Immunohistochemistry successfully uncovers Intratumoral heterogeneity and widespread co-losses of chromatin regulators in clear cell renal cell carcinoma. PLoS One. 2016; 11(10):e0164554

33. Wang $M$, Zhao J, Zhang L, Wei F, Lian Y, Wu Y, Gong Z, Zhang S, Zhou J, Cao K, et al. Role of tumor microenvironment in tumorigenesis. J Cancer. 2017:8(5):761-73.

34. Fang M, Yuan J, Peng C, Li Y. Collagen as a double-edged sword in tumor progression. Tumour Biol. 2014;35(4):2871-82.

35. Bhola NE, Grandis JR. Crosstalk between G-protein-coupled receptors and epidermal growth factor receptor in cancer. Front Biosci. 2008;13:1857-65.

36. Lappano R, Maggiolini M. G protein-coupled receptors: novel targets for drug discovery in cancer. Nat Rev Drug Discov. 2011;10(1):47-60.

37. Nieto Gutierrez A, McDonald PH. GPCRs: emerging anti-cancer drug targets. Cell Signal. 2018;41:65-74.

38. Hauser AS, Attwood MM, Rask-Andersen M, Schioth HB, Gloriam DE. Trends in GPCR drug discovery: new agents, targets and indications. Nat Rev Drug Discov. 2017;16(12):829-42.

39. Lu B, Sun X, Chen Y, Jin Q, Liang Q, Liu S, Li Y, Zhou Y, Li W, Huang Z. Novel function of PITH domain-containing 1 as an activator of internal ribosomal entry site to enhance RUNX1 expression and promote megakaryocyte differentiation. Cell Mol Life Sci. 2015;72(4):821-32.

40. Wang XQ, Tang ZX, Yu D, Cui SJ, Jiang YH, Zhang Q, Wang J, Yang PY, Liu F. Epithelial but not stromal expression of collagen alpha-1(III) is a diagnostic and prognostic indicator of colorectal carcinoma. Oncotarget. 2016;7(8):8823-38.

41. Sun Q, Zhao H, Zhang C, Hu T, Wu J, Lin X, Luo D, Wang C, Meng L, Xi L, et al. Gene co-expression network reveals shared modules predictive of stage and grade in serous ovarian cancers. Oncotarget. 2017;8(26):42983-96.

42. Januchowski R, Swierczewska M, Sterzynska K, Wojtowicz K, Nowicki M, Zabel M. Increased expression of several collagen genes is associated with drug resistance in ovarian Cancer cell lines. J Cancer. 2016;7(10):1295-310.
43. Xiong G, Deng L, Zhu J, Rychahou PG, Xu R. Prolyl-4-hydroxylase alpha subunit 2 promotes breast cancer progression and metastasis by regulating collagen deposition. BMC Cancer. 2014;14:1.

44. Brisson BK, Mauldin EA, Lei W, Vogel LK, Power AM, Lo A, Dopkin D, Khanna C, Wells RG, Pure E, et al. Type III collagen directs stromal organization and limits metastasis in a murine model of breast Cancer. Am J Pathol. 2015; 185(5):1471-86.

45. Patel N, Itakura $T$, Jeong $S$, Liao CP, Roy-Burman $P$, Zandi E, Groshen $S$, Pinski J, Coetzee GA, Gross ME, et al. Expression and functional role of orphan receptor GPR158 in prostate cancer growth and progression. PLoS One. 2015;10(2):e0117758.

46. Li N, Zhang Y, Sidlauskas K, Ellis M, Evans I, Frankel P, Lau J, El-Hassan T, Guglielmi L, Broni J, et al. Inhibition of GPR158 by microRNA-449a suppresses neural lineage of glioma stem/progenitor cells and correlates with higher glioma grades. Oncogene. 2018;37(31):4313-33.

47. Zhang LQ, Yang SQ, Qu XD, Chen XJ, Lu HS, Wang Y. GRP137 promotes cell proliferation and metastasis through regulation of the PI3K/AKT pathway in human ovarian cancer. Tumori. 2018;104(5):330-7.

48. Zhu CX, Xiong W, Wang ML, Yang J, Shi HJ, Chen HQ, Niu G. Nuclear G protein-coupled oestrogen receptor (GPR30) predicts poor survival in patients with ovarian cancer. J Int Med Res. 2018;46(2):723-31.

49. Liu Z, Huang Z, Yang W, Li Z, Xing S, Li H, Hu B, Li P. Expression of orphan GPR56 correlates with tumor progression in human epithelial ovarian cancer. Neoplasma. 2017;64(1):32-9.

\section{Publisher's Note}

Springer Nature remains neutral with regard to jurisdictional claims in published maps and institutional affiliations.
Ready to submit your research? Choose BMC and benefit from:

- fast, convenient online submission

- thorough peer review by experienced researchers in your field

- rapid publication on acceptance

- support for research data, including large and complex data types

- gold Open Access which fosters wider collaboration and increased citations

- maximum visibility for your research: over $100 \mathrm{M}$ website views per year

At BMC, research is always in progress.

Learn more biomedcentral.com/submissions 